\title{
Crystallographic Orientation-Aligned ZnO Nanorods Grown by a Tin Catalyst
}

2003

Vol. 3, No. 9

$1315-1320$

\author{
P. X. Gao, Y. Ding, and Z. L. Wang* \\ School of Materials Science and Engineering, Georgia Institute of Technology, \\ Atlanta, Georgia 30332-0245
}

Received July 21, 2003; Revised Manuscript Received July 30, 2003

\begin{abstract}
Well-aligned $\mathrm{ZnO}$ nanorods with identical crystallographic orientation have been synthesized using a vapor transport deposition process. Orientation-ordered nanorods grow normal to the $c$ planes of the as-deposited micrometer-sized $\mathrm{ZnO}$ rods on a polycrystalline $\mathrm{Al}_{2} \mathrm{O}_{3}$ substrate, and each nanorod is along [0001] and enclosed by $\{2 \overline{1} \overline{10}\}$ facet surfaces. The nanorods remain in an identical crystal orientation with a homoepitaxial orientation relationship with the microrod. During the synthesis, reduced $\mathrm{Sn}$ from $\mathrm{SnO}_{2}$ powder added to the source materials functions as a catalyst, guiding the orientation-aligned growth of $\mathrm{ZnO}$ nanorods. By controlling the growth time at high temperature, uniform lengths of aligned nanorods have been received. This work demonstrates that metallic $\mathrm{Sn}$ could be a good candidate for catalyzing the growth of $1 \mathrm{D}$ nanostructures.
\end{abstract}

Zinc oxide, a wide band gap (3.37 eV) semiconducting, piezoelectric, and photoconducting material, has a wide range of applications in solar cells, ${ }^{1}$ sensors, ${ }^{2-4}$ optoelectronic devices, and surface acoustic waveguides. ${ }^{5}$ Recently, 1D nanostructures of $\mathrm{ZnO}$, including nanowires,${ }^{6}$ nanobelts, ${ }^{7}$ and nanotubes, ${ }^{8}$ have been attracting vast interest in the field of nanotechnology. Hierarchy and aligned $\mathrm{ZnO}$ nanostructures are finding potential applications in lasers, ${ }^{6}$ sensors,,${ }^{9} 10$ lightemitting diodes, ${ }^{11}$ nanoresonators and cantilevers, ${ }^{12,13}$ and field-emission devices. ${ }^{14-16}$

In syntheses of aligned $1 \mathrm{D} \mathrm{ZnO}$ nanostructures, catalysts such as $\mathrm{Au},{ }^{6,17} \mathrm{Co},{ }^{15}$ and $\mathrm{NiO}^{18}$ have been introduced into the synthesis process to guide the oriented growth of $\mathrm{ZnO}$ nanowires, and aligned growth without catalysis has also been achieved using low-temperature CVD (chemical vapor deposition) ${ }^{19}$ and ultrahigh vacuum deposition techniques such as MOCVD (metal-organic chemical vapor deposition) ${ }^{11,14}$ and MOVPE (metal-organic vapor-phase epitaxy). ${ }^{20}$ In this letter, we report a novel method of synthesizing orientation-aligned $\mathrm{ZnO}$ nanorods and nanobelts using metallic $\mathrm{Sn}$ as a catalyst. The $\mathrm{ZnO}$ nanorods have an identical crystallographic orientation, and their lengths have been controlled via the deposition time.

A chemical vapor transport process ${ }^{6,17,21-23}$ was used for the syntheses of aligned $\mathrm{ZnO}$ nanorods and nanobelts. The experimental apparatus includes a horizontal tube furnace, a rotary pump system, and a gas supply system. A mixture of commercial $\mathrm{ZnO}, \mathrm{SnO}_{2}$, and graphite powders in a certain ratio $(2: 1: 1 \mathrm{Zn} / \mathrm{Sn} / \mathrm{C})$ was placed in an alumina boat as the source material and positioned at the center of the alumina

\footnotetext{
* Corresponding author. E-mail: zhong.wang@mse.gatech.edu.
}

tube. To investigate the duration time dependence on the growth of desired nanostructures, we conducted the deposition process at $1150{ }^{\circ} \mathrm{C}$ for $5,15,30$, and 60 min under a constant pressure of 200 mbar and an Ar flow rate of 20 sccm (standard cubic centimeters per minute). The entire length of the tube furnace is $50 \mathrm{~cm}$. The desired nanostructures were deposited onto an alumina substrate located 21 $\mathrm{cm}$ away from the center of the furnace in a temperature range of $550-600{ }^{\circ} \mathrm{C}$. Morphological, chemical, and crystallographic characterizations of the as-prepared samples were carried out using scanning electron microscopy (SEM), transmission electron microscopy (TEM), and electron dispersive X-ray spectroscopy (EDS).

Figure 1 is a group of typical SEM images of the as-synthesized nanostructures on an alumina substrate after 5 min of deposition at $1150{ }^{\circ} \mathrm{C}$, showing the initial growth characteristics of the desired nanostructure. Micrometer-sized $\mathrm{ZnO}$ rods (microrods) of irregular side surfaces but uniform flat (0001) surfaces of $\sim 3 \mu \mathrm{m}$ are formed on the alumina substrate, which sometime show faceted side surfaces (Figure 1b). On each microrod, there are a number of nanorods oriented perpendicular to the (0001) plane, as were clearly depicted in Figure 1c and d, which are respectively the top and side views of the oriented $\mathrm{ZnO}$ nanorods. At the tip of each nanorod and nanobelt, there is a comparably sized $\mathrm{Sn}$ ball. The oriented nanorods dispersively distribute on the flat $c$ plane of the microrods with uniform diameters ranging from $20-40 \mathrm{~nm}$ and an average height of about $40-80 \mathrm{~nm}$.

Upon extending the growth time at $1150{ }^{\circ} \mathrm{C}$ to $15 \mathrm{~min}$, it is shown in Figure 2 that the oriented $\mathrm{ZnO}$ nanorods get longer and some nanobelts grow out of the side surfaces of 


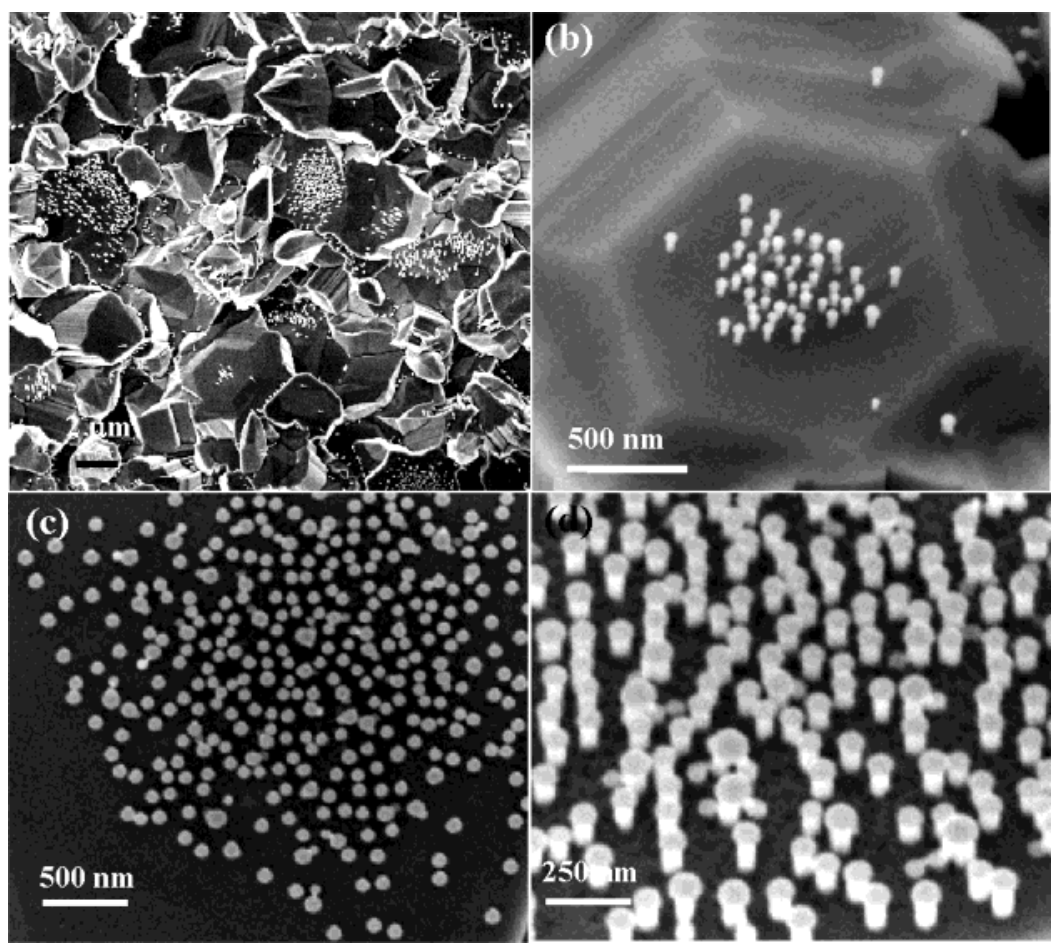

Figure 1. Initial growth of oriented nanorods using metallic $\mathrm{Sn}$ as the catalyst at $1150{ }^{\circ} \mathrm{C}$ with a growth time of 5 min. (a) Micrometerscale $\mathrm{ZnO}$ short rods rooted on an $\mathrm{Al}_{2} \mathrm{O}_{3}$ substrate with aligned nanorods dispersively distributed on the top surfaces of the microrods. (b) Hexagonally faceted $\mathrm{ZnO}$ microrod with aligned $\mathrm{ZnO}$ nanorods on its top (0001) surface. (c, d) Top and side views, respectively, of the oriented $\mathrm{ZnO}$ nanorods on the top of a single-crystal microrod.

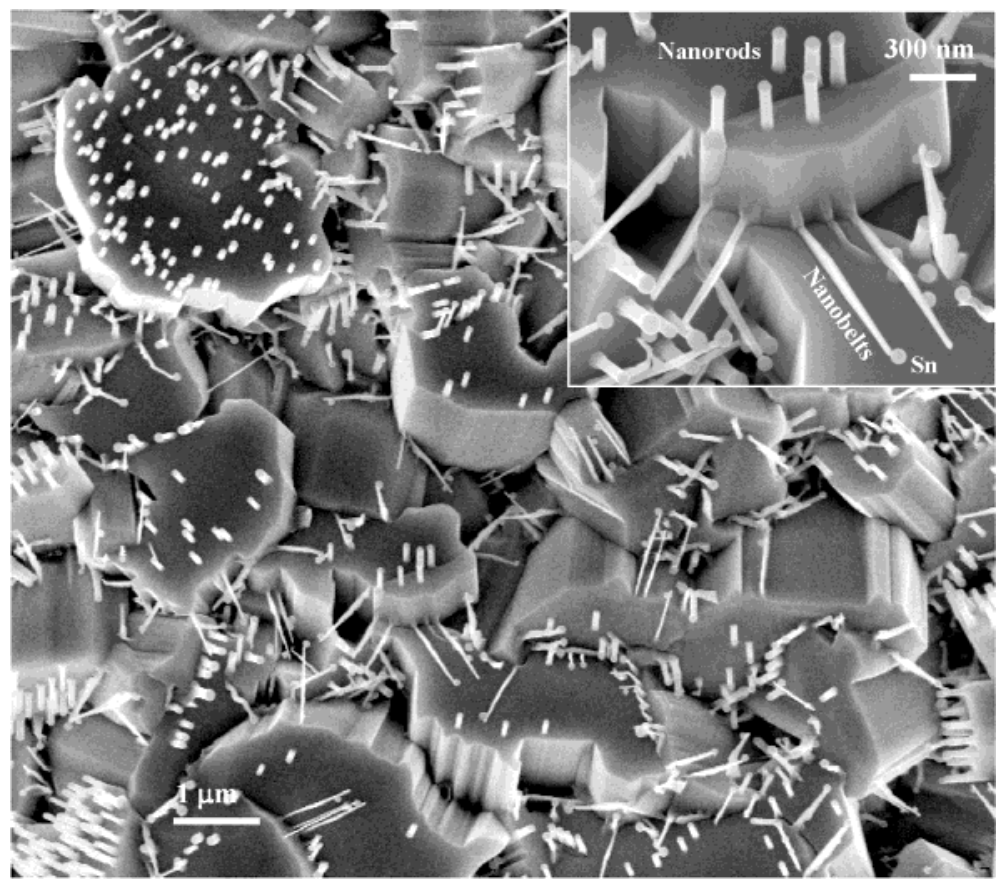

Figure 2. $\mathrm{ZnO}$ nanorods and nanobelts grown on the top and side surfaces of the $\mathrm{ZnO}$ microrods, respectively, after extending the growth time to $15 \mathrm{~min}$. (Inset) Magnified SEM image.

the microrods. A higher-magnification picture clearly describes the perpendicular directional growth of the nanobelts (as indicated in the inset of Figure 2), where the nanorods have dimensions of $30-50 \mathrm{~nm}$ in diameter and $100-200$ $\mathrm{nm}$ in length and nanobelts have dimensions of $10-30 \mathrm{~nm}$ in width and 300-500 $\mathrm{nm}$ in length.
The data suggest that the diameters of the nanorods are well confined by the size of the metallic Sn ball on the tip and that the growth time has a little influence on the diameter of the nanorods. Parts a and $b$ of Figure 3 give a good description of the oriented growth of the nanorods on the $c$ plane of the $\mathrm{ZnO}$ microrods. A closer examination of the 


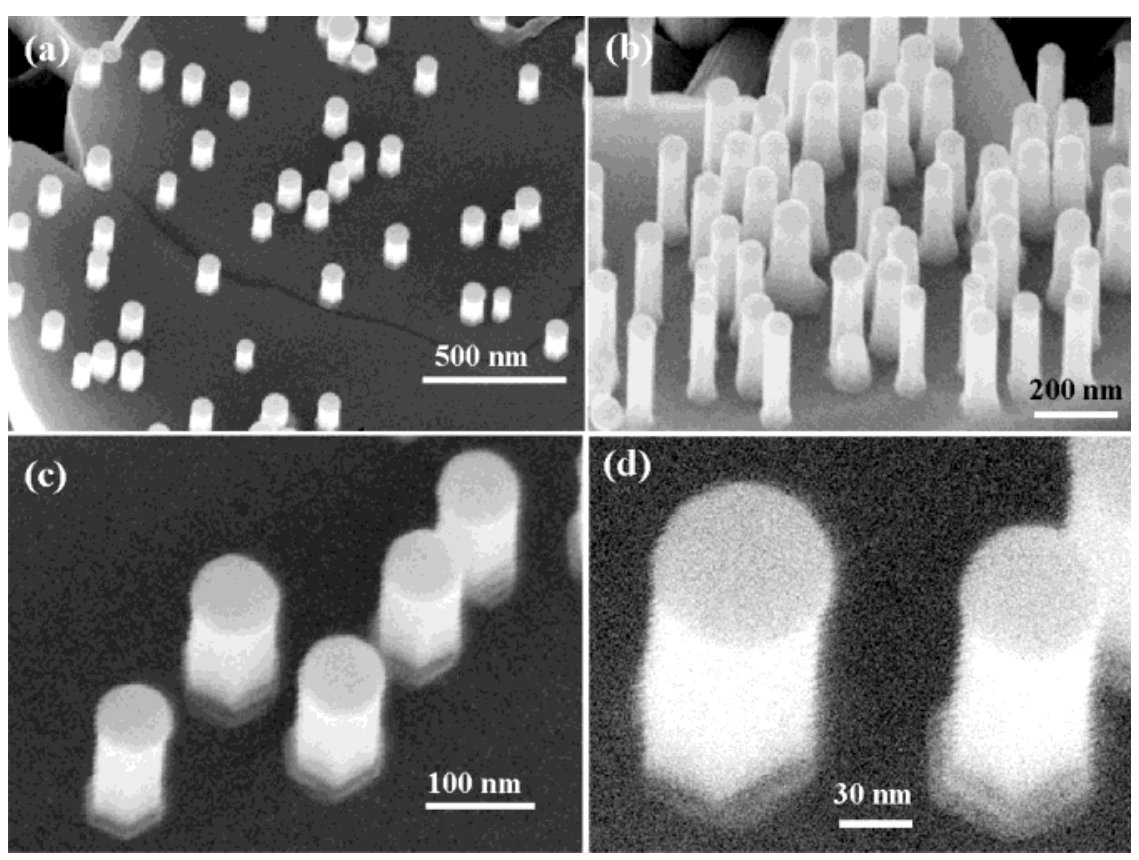

Figure 3. Perpendicular growth of nanorods on the $c$ plane of $\mathrm{ZnO}$ microrods. $(\mathrm{a}, \mathrm{b})$ Dispersively oriented nanorods with inter-rod distances of $\sim 400$ and $\sim 100 \mathrm{~nm}$, respectively. (c, d) Epitaxial growth of the $\mathrm{ZnO}$ nanorods, forming arrays with an identical crystallographic orientation, as represented by the parallel side surfaces.

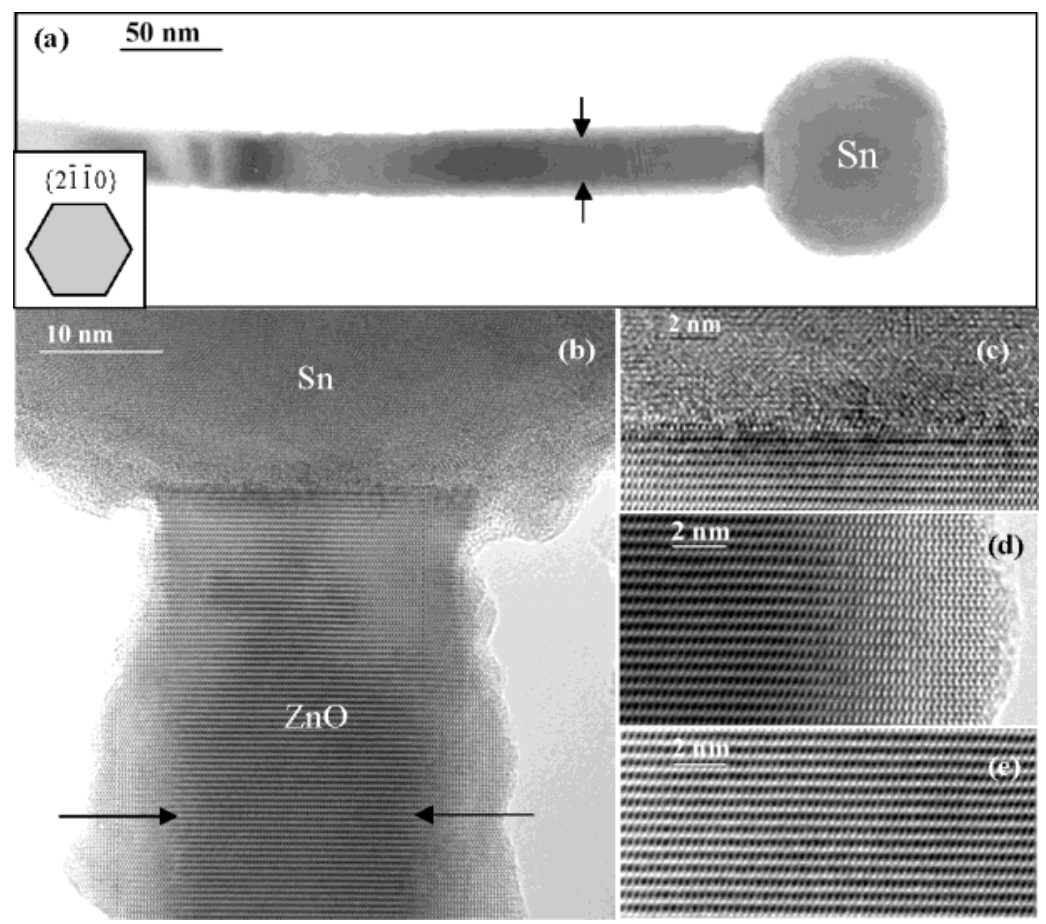

Figure 4. (a) Low-magnification TEM image of a $\mathrm{ZnO}$ nanorod with a model of its cross section in the inset. (b) High-resolution TEM image of the nanorod with the incident electron beam along [2110]. (c-e) Enlarged images from the $\mathrm{Sn}-\mathrm{ZnO}$ interface, the edge of the nanorod, and the central region of the nanorod, respectively.

root of each nanorod in Figure $3 \mathrm{c}$ and $\mathrm{d}$ shows that each nanorod tends to grow along the same crystallographic orientation as the microrod with six parallel faceted surfaces of $\{2 \overline{1} \overline{1} 0\}$, which indicates an orientation alignment of the $\mathrm{ZnO}$ nanorods and a coherent epitaxial relationship with the base $\mathrm{ZnO}$ microrod.

Transmission electron microscopy imaging of a $\mathrm{ZnO}$ nanorod is presented in Figure 4. An Sn ball with a faceted surface $^{24}$ is located at the growth front (Figure 4a). A highresolution TEM image presented in Figure $4 \mathrm{~b}$ shows that the $\mathrm{ZnO}$ nanorod grows along [0001] and that its volume is free from dislocation. The interface between the $\mathrm{Sn}$ particle and the $\mathrm{ZnO}$ nanorod is atomically sharp and there may be a coherent relationship between the atomic lattices at the interface (Figure 4c), but the $\mathrm{Sn}$ is not well crystallized. From the image contrast across the nanorod presented in the low- 

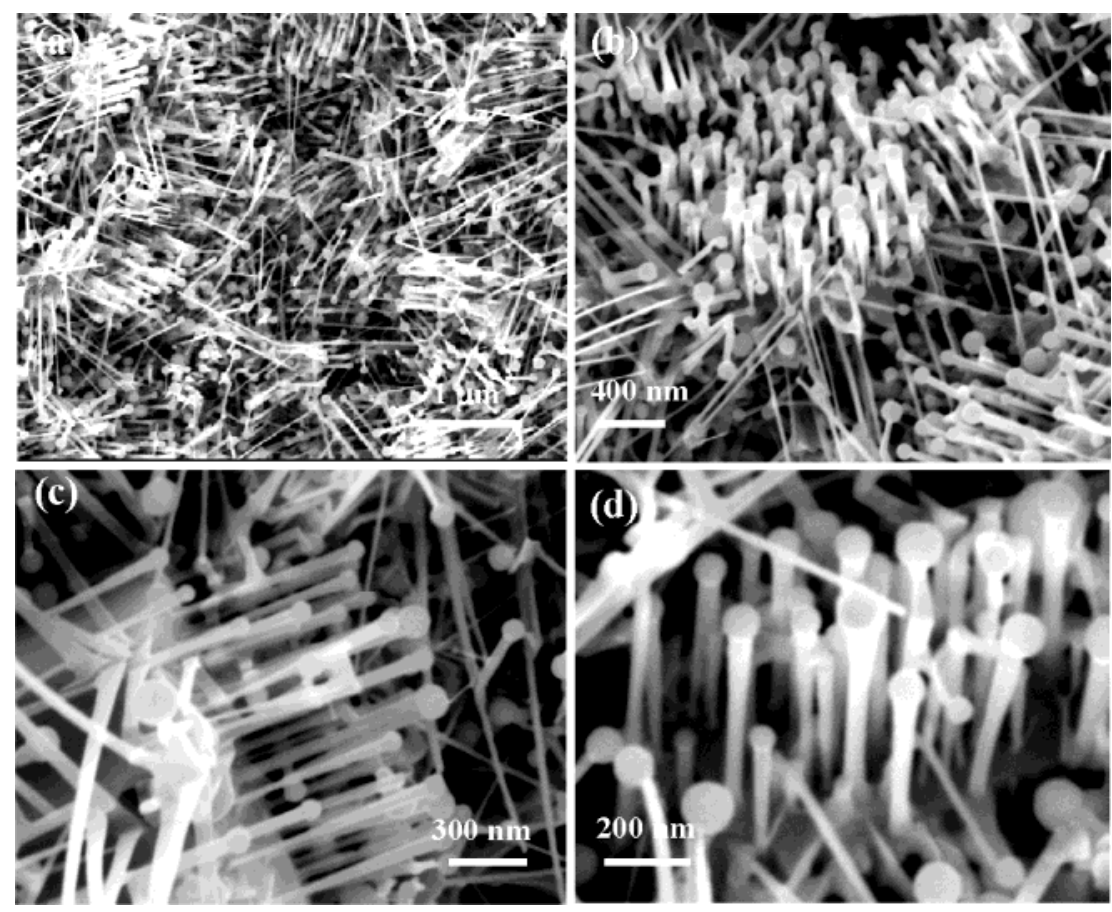

Figure 5. Typical morphology of the as-synthesized nanorods and nanobelts after 60 min of growth at $1150{ }^{\circ} \mathrm{C}$. (a) Aligned $\mathrm{ZnO}$ nanorods and side-branched nanobelts on microrods. (b, c) Perpendicular orientation relationship between the oriented $\mathrm{ZnO}$ nanorods and the surrounding nanobelts on a single microrod as viewed from the top and the side, respectively. (d) Magnified view of the oriented nanorods rooted on a microrod.

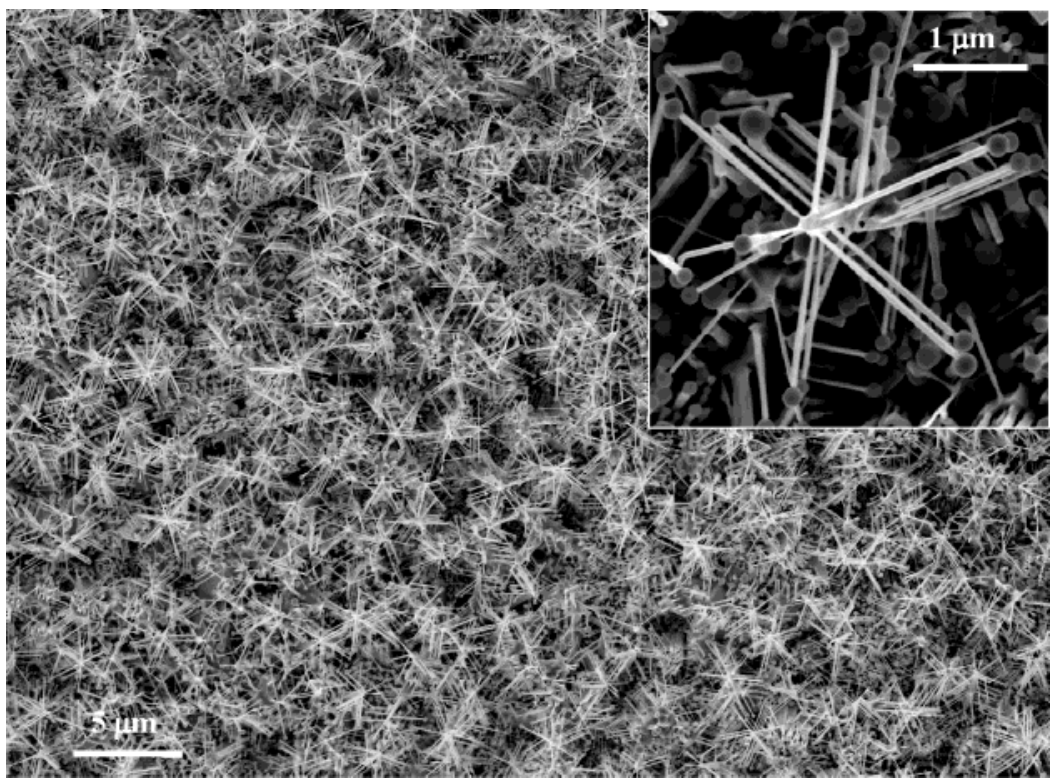

Figure 6. Top view of the well-aligned nanowire-nanobelt junction arrays on an alumina substrate. (Inset) Magnified micrograph of a single set of junction arrays with a 6-fold-symmetric structural feature.

magnification image (Figure 4a) and in the high-magnification TEM image in Figure 4b, a uniform contrast region at the middle of the nanorod (see the region indicated by arrows in Figure $4 b$ and the enlarged image in Figure 4e) indicates its constant projected thickness, and the side edges are very thin (Figure 4d). On the basis of the image information, the six side surfaces of the nanorods are defined to be $\{2 \overline{1} 10\}$, and the electron beam direction is [211ㅣ]. A schematic model of the nanorod cross section is inserted into Figure $4 \mathrm{a}$.
With increasing growth time, the oriented nanorods and nanobelts grew longer and longer. Figure 5 shows a typical morphology of the as-synthesized nanostructructures upon $60 \mathrm{~min}$ at $1150{ }^{\circ} \mathrm{C}$. Figure $5 \mathrm{a}$ shows the as-deposited nanostructures composed of the orientation-aligned $\mathrm{ZnO}$ nanorods and nanobelts out of the top and side surfaces of the microrods (Figure $5 \mathrm{~b}$ and $\mathrm{c}$ ). The $\mathrm{ZnO}$ nanorods grow out of the $c$ plane with a uniform length of $0.5-1.0 \mu \mathrm{m}$, and the diameter remains around $30-60 \mathrm{~nm}$. Compared to 
those grown for a shorter time and separated by $100 \mathrm{~nm}-1$ $\mu \mathrm{m}$, these oriented nanorods grew densely with a separation of 50-150 nm, suggesting an increase in the number of nucleation sites during the growth. A slight increase in the diameter at the upper part of the nanorod is likely due to an increase in the size of the Sn catalyst particle during the growth. High-magnification images (Figure $5 b$ and d) show that the microrods are rougher than those grown for $5 \mathrm{~min}$. Along the perpendicular directions to the oriented nanorods, the number of side-branched nanobelts of a uniform length of $1 \mu \mathrm{m}$ also dramatically increases. It is interesting that the metallic Sn head on the top of the nanorod is about onethird larger in diameter than the nanorod diameter and that the size of the Sn head on a nanobelt is about 2 to 3 times larger than the width of the nanobelt.

To study the effect of graphite as a vapor-generation agent for the growth of the desired nanostructures, an experimental session was conducted by adjusting the molar ratio of corresponding $\mathrm{Zn} / \mathrm{Sn} / \mathrm{C}$ from 2:1:1 to 2:1:2 while keeping the other parameters the same as for the experiments presented above and limiting the growth time to $1 \mathrm{~h}$. Surprisingly, well-aligned nanowire-nanobelt junction arrays $^{21}$ formed perpendicularly oriented on the alumina substrate. Figure 6 is a top view of the as-grown junction arrays over a large area, where the 6-fold-symmetric feature of each junction array is clearly seen. The inset is a typical magnified micrograph of a single junction array. Similar to the structure reported previously, ${ }^{21}$ the junction array consists of a central nanowire $100-200 \mathrm{~nm}$ in diameter and is surrounded by 6 -fold-symmetric nanobelts $50-100 \mathrm{~nm}$ in width that grow along the six crystallographic equivalent directions of $\langle 01 \overline{1} 0\rangle$.

Graphite is introduced into the raw material to increase the creation rate of $\mathrm{Zn}$ and $\mathrm{Sn}$ vapors. Carbon can reduce $\mathrm{ZnO}$ and $\mathrm{SnO}_{2}$ in $\mathrm{Zn}$ and $\mathrm{Sn}$ vapors at relatively low temperature. It is expected that doubling the content of graphite in the raw material will increase the concentration of Sn liquid droplets, giving rise to many more nucleation sites $^{25}$ and leading to the growth of high-density nanorods and nanobelts. According to previous studies on junction arrays, ${ }^{21}$ it is known that the side-branched nanobelts would like to grow along six crystallographically equivalent orientations $\langle 01 \overline{1} 0\rangle$, giving 6-fold-symmetric nanowire-nanobelt junction arrays presented in Figure 6 .

To optimize the desired morphology of the oriented $\mathrm{ZnO}$ nanorods, we tested some more parameters. Figure 7 shows a typical morphology of the as-prepared nanorods and nanobelts after $30 \mathrm{~min}$ of growth at $1100{ }^{\circ} \mathrm{C}$, where we used only two-thirds the amount of $\mathrm{SnO}_{2}$ used in the other experiments and kept the pressure and argon flow rate the same. In Figure 7a, it is clearly shown that, in addition to the oriented $\mathrm{ZnO}$ nanorods, there are also some $\mathrm{ZnO}$ belt networks normal to and between the oriented nanorods, as indicated by arrows. These networks are parallel to the nanobelts directly growing from the side surfaces of the microrod base because of the homoepitaxial orientation relationship. ${ }^{26}$ Figure $7 \mathrm{~b}$ is a magnified view of the vertical crystallographic orientation-aligned $\mathrm{ZnO}$ nanorods on a $\mathrm{ZnO}$

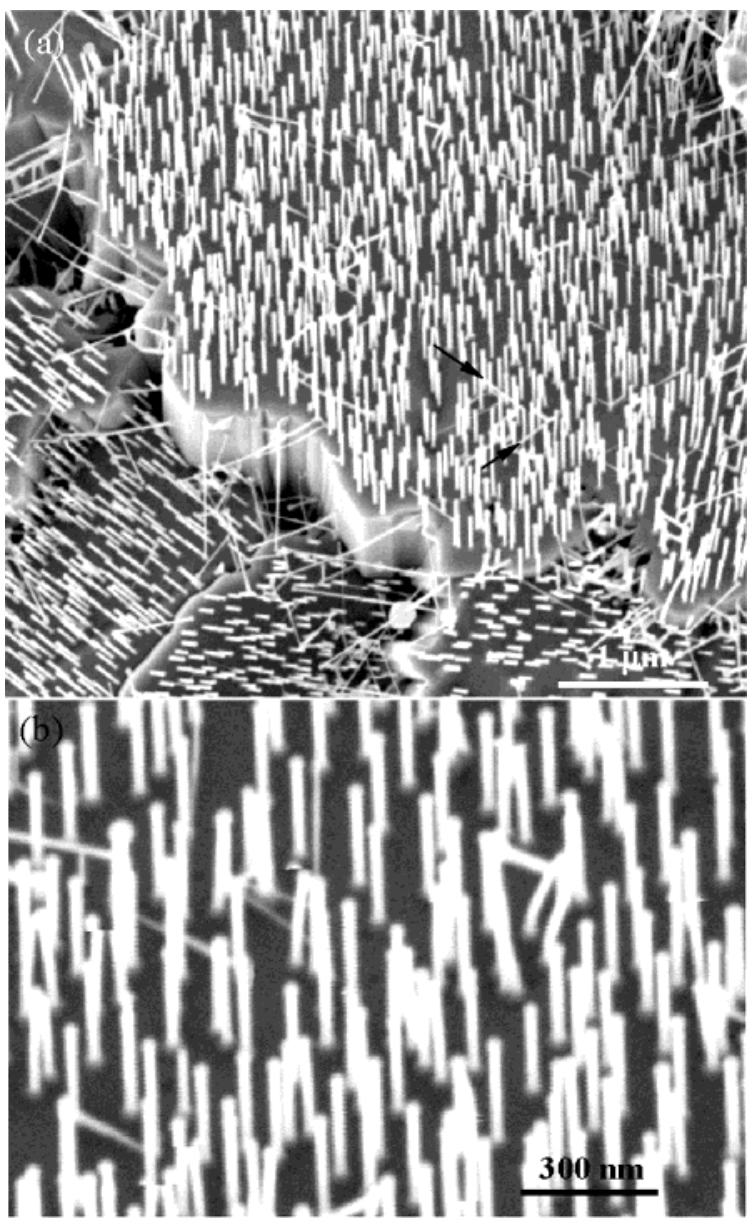

Figure 7. Typical morphology of the as-synthesized nanorods and nanobelts after $30 \mathrm{~min}$ of growth at $1100{ }^{\circ} \mathrm{C}$. (a) Well-aligned nanorods on a larger surface area. Nanobelts grown out of the nanorods in a perpendicular direction are also identified, as indicated by arrows. The alignment in the nanobelt directions indicates the crystallographic-orientation ordering among the nanorods. (b) Magnified view of the crystallographically aligned $\mathrm{ZnO}$ nanorod arrays.

microrod, displaying a uniform diameter of $\sim 25 \mathrm{~nm}$ and a height of $\sim 250 \mathrm{~nm}$.

There are two processes for the growth of $\mathrm{ZnO}$ nanorods. One is the oxidation of the reduced and vaporized $\mathrm{Zn}$ following the vapor-liquid-solid (VLS) growth process, ${ }^{6,21,27}$ and the other is the direct deposition of the $\mathrm{ZnO}$ vapor (the so-called vapor-solid (VS) process) ${ }^{7}$. On the basis of our experimental data presented here and published previously, ${ }^{28,29}$ reductions of $\mathrm{ZnO}$ and $\mathrm{SnO}_{2}$ in $\mathrm{Zn}$ and $\mathrm{Sn}$ vapors, respectively, are possible in the high-temperature zone. The carrier gas transfers the reduced and vaporized $\mathrm{Zn}$ and $\mathrm{Sn}$ to the lower-temperature region. The oxidation of $\mathrm{Zn}$ vapor and its subsequent deposition onto the substrate form single-crystal $\mathrm{ZnO}$ microrods with flat (0001) surfaces. Simultaneously, $\mathrm{Sn}$ vapor would be transformed to liquid droplets falling down onto the surface of the $\mathrm{ZnO}$ microrods. Then, on these flat planes, dispersed metallic Sn droplets function as catalysts to guide the oriented growth of $\mathrm{ZnO}$, which in fact is a homoepitaxial process with the bulk $\mathrm{ZnO}$ microrods. ${ }^{26}$ The oriented nanorods remain in an identical orientation with the microrods, resulting in the crystal- 
lographic orientation-aligned growth of the nanorods on the (0001) surface. However, the liquid tin droplets in contact with the side surface of the $\mathrm{ZnO}$ microrods guide the growth of $\mathrm{ZnO}$ nanobelts along $\langle 01 \overline{1} 0\rangle$ perpendicular to the $\mathrm{ZnO}$ nanorods. A continuous deposition of Sn vapor at the growth front results in a continuous increase in the size of the $\mathrm{Sn}$ particle, leading to a continuous increase in the size of the nanorods/nanobelts as growth proceeds.

Our data suggest that the VLS process is dominant and the VS process is supplementary. As presented in Figure 1d, the microrods as the growth platforms are quite rough at the very beginning between the oriented nanorods and even form deep notches as if they were etched off at longer growth times. Then it is expected that, as the growth time increases, the source materials are consumed, the supply of vapors is short, and the vapor pressure may fall below the supersaturation threshold required for VLS growth. The growth can be continued only by vaporizing the microrods below the nanorods, resulting in the deep notches on microrod surfaces.

In summary, by using a simple vapor-transport deposition method, well-aligned $\mathrm{ZnO}$ nanorods with six identical facets of $\{2 \overline{1} \overline{1} 0\}$ and orientation ordering have been successfully synthesized. Reduced $\mathrm{Sn}$ from $\mathrm{SnO}_{2}$ acts as a catalyst that guides the aligned growth of $\mathrm{ZnO}$ nanorods. By controlling the growth time at high temperature, nanorods of uniform lengths have been produced. By increasing the content of graphite as the vapor-generating agent, well-aligned nanowire-nanobelt junction arrays have also been received. This study demonstrates that $\mathrm{Sn}$ can be an effective catalyst that can be used to grow crystallographic orientation-aligned nanorods.

Acknowledgment. We are grateful for financial support from the NASA URETI program.

\section{References}

(1) Keis, K.; Vayssieres, L.; Lindquist, S.; Hagfeldt, A. Nanostruct. Mater. 1999, 12, 487.

(2) Minne, S. C.; Manalis, S. R.; Quate, C. F. Appl. Phys. Lett. 1995, 67, 3918.
(3) Shibata, T.; Unno, K.; Makino, E.; Ito, Y.; Shimada, S. Sens. Actuators, A 2002, 102, 106.

(4) Lin, H. M.; Tzeng, S. J.; Hsiau, P. J.; Tsai, W. L. Nanostruct. Mater. 1999, 10, 465

(5) Gorla, C. R.; Emanetoglu, N. W.; Liang, S.; Mayo, W. E.; Lu, Y.; Wraback, M.; Shen, H. J. Appl. Phys. 1999, 85, 2595.

(6) Huang, M. H.; Mao, S.; Feick, H.; Yan, H. Q.; Wu, Y. Y.; Kind, H.; Weber, E.; Russo R.; Yang, P. D. Science 2001, 292, 1897.

(7) Pan, Z. W.; Dai Z. R.; Wang, Z. L. Science 2001, 291, 1947.

(8) Wu, J. J.; Liu, S. C.; Wu, C. T.; Chen, K. H.; Chen, L. C. Appl. Phys. Lett. 2002, 81, 1312.

(9) Arnold, M. S.; Avouris, P.; Pan, Z. W.; Wang, Z. L. J. Phys. Chem. B 2003, 107, 659 .

(10) Comini, E.; Faglia, G.; Sberveglieri, G.; Pan, Z. W.; Wang, Z. L. Appl. Phys. Lett. 2003, 81, 1869.

(11) Park, W. I.; Yi, G. C.; Kim, J. W.; Park, S. M. Appl. Phys. Lett. 2003, 82, 4358.

(12) Bai, X. D.; Gao, P. X.; Wang, Z. L.; Wang, E. G. Appl. Phys. Lett. 2003, 82, 4806.

(13) Hughes, W.; Wang, Z. L. Appl. Phys. Lett. 2003, 82, 2886.

(14) Park, W. I.; Yi, G.; Kim, M.; Pennycook, S. L. Adv. Mater. 2002, $14,1841$.

(15) Lee, C. J.; Lee, T. J.; Lyu, S. C.; Zhang, Y.; Ruh, H.; Lee, H. J. Appl. Phys. Lett. 2002, 19, 3648.

(16) Bai, X. D.; Wang, E. G.; Gao, P. X.; Wang, Z. L. Nano Lett., in press, 2003.

(17) Ng, H. T.; Li, J.; Smith, M. K.; Nguyen, P.; Cassell, A.; Han, J.; Meyyanppan, M. Science 2003, 300, 1249.

(18) Lyu, S. C.; Zhang, Y.; Ruh, H.; Lee, H. J.; Shim, H. W.; Suh, E. K.; Lee, C. J. Chem. Phys. Lett. 2002, 363, 134.

(19) Wu, J.-J.; Liu, S.-C. Adv. Mater. 2002, 14, 215.

(20) Ogata, K.; Maejima, K.; Fujita, Sz.k; Fujita, Sg. J. Cryst. Growth 2003, 248, 25.

(21) Gao,P. X.; Wang, Z. L. J. Phys. Chem. B 2002, 106, 12653.

(22) Lao, J. Y.; Wen, J. G.; Ren, Z. F. Nano Lett. 2002, 2, 1287.

(23) Hassani, S.; Tromson-Carli, A.; Lusson, A.; Didier, G.; Triboulet, R. Phys. Status Solidi B 2002, 229, 835.

(24) Zhu, Y. Q.; Hsu, W. K.; Zhou, W. Z.; Terrones, M.; Kroto, H. W.; Walton, D. R. M. Chem. Phys. Lett. 2001, 347, 337.

(25) Abduev, A. K.; Akhmedov, A. K.; Baryshnikov, V. G.; Shakhshaev, Sh. O. Tech. Phys. Lett. 2000, 4, 37.

(26) Ogata, K.; Kawanishi, T.; Maejima, K.; Fujita, Sz.; Fujita, Sg. J. Cryst. Growth 2002, 237-239, 553.

(27) Wagner, R. S.; Ellis, W. C. Appl. Phys. Lett. 1964, 4, 89.

(28) Dai, Z. R.; Pan, Z. W.; Wang, Z. L. J. Am. Chem. Soc. 2002, 124, 8673.

(29) Gao, P. X.; Wang, Z. L. J. Am. Chem. Soc., in press, 2003.

NL034548Q 